\title{
On the limits of added-mass theory in separated flows and with varying initial conditions
}

\author{
J. N. Fernando, G. D. Weymouth and D. E. Rival \\ Department of Mechanical and Materials Engineering, Queen's University, Kingston, ON \\ $K^{\prime} 7$ L 3 6, Canada \\ Southampton Marine and Maritime Institute, University of Southampton, Southampton \\ SO17 1BJ, UK
}

\begin{abstract}
It remains unclear to what extent inviscid added-mass theory accounts for the forces exerted on an accelerating body subjected to separated flow. In this study, reactant forces and velocity-field data are systematically acquired using experimental measurements and simulations of an accelerating circular flat plate. Cases accelerated from rest are compared to cases accelerated from a steady flow state. When the added-mass forces predicted by potential theory and the resistance forces associated with the instantaneous plate velocity are accounted for, the remaining (residual) forces comprise approximately $20 \%$ of the peak force, even at high accelerations. In addition, the computed residual forces during accelerations both from rest and steady-state cases yield good collapse with respect to one another, indicating that the total forces are not a strong function of the initial state of the wake. These results suggests that inviscid added-mass theory is inadequate to predict the full reactant force even in the 'ideal' condition of impulsive motion from rest.
\end{abstract}

Keywords: Separated flows, swimming/flying, vortex dynamics

\section{Introduction}

Acceleratory motions are a ubiquitous feature of natural propulsion and are an important consideration in the design of many onshore and offshore structures as well. Offshore platform motions in particular have received much attention, and are the topic of investigations spanning many years (see for instance the comprehensive study by Sarpkaya and Isaacson (1981), 
where the current understanding of the forces in viscous flows are reviewed). The forces that arise when fluid accelerates around a body have classically been resolved into added-mass, viscous-drag, and flow-history contributions (Odar and Hamilton, 1964; Karanfilian and Kotas, 1978; Clift et al., 1978). The added-mass force, a concept derived from inviscid theory, has been the subject of numerous studies in recent years, primarily due to its relevance to propulsion and manoeuvrability (Weymouth and Triantafyllou, 2012, 2013; Polet et al., 2015). Determining whether added-mass is sufficient to account for reactant forces on bodies subjected to accelerations with separated flow is therefore of great practical interest.

Classical aerodynamic models proposed by Theodorsen (1935) and Küssner (1936), for instance, have prompted a contemporary force decomposition involving circulatory and non-circulatory (added-mass) effects. A few recent studies, such as Baik et al. (2012), have applied such a decomposition to the force histories of flat plates accelerating from rest. Pitt Ford and Babinsky (2013) predicted the non-circulatory lift on an impulsively-started flat plate at an angle of attack of $15^{\circ}$ using a two-dimensional potential flow model coupled with values for the bound circulation that yielded the best agreement with the experimental measurements. They showed that the lift force produced by estimates of added mass was responsible for nearly half the lift during the acceleration period. The remaining lift force was attributed to circulation build-up in shed vortices, rather than bound circulation. Mancini et al. (2015) performed a similar analysis on surging flat plates at large angles of attack using the solution for the added-mass derived by Pitt Ford and Babinsky (2013) to determine the relative contributions of both the circulatory and non-circulatory effects. However, most studies have investigated reactant force decomposition for accelerations from rest (Prandtl and Tietjens, 1957; Manar et al., 2015; Babinsky et al., 2016), giving little attention to conditions in which the wake may already be strongly rotational. In addition, the argument in Leonard and Roshko (2001) extending potential-flow added-mass to general viscous flows hinges on a slip velocity applied at the body boundary, a concept which only applies in the limit of impulsive accelerations. While this may account for the need for a circulatory reactant force at finite accelerations, it has not been shown that this force goes to zero as the acceleration magnitude increases.

As such, the source of instantaneous forces exerted on bodies undergoing general acceleratory motions in a highly separated flow is still unresolved. To this end, this investigation acts as a benchmark study by providing an 
empirical decomposition of the forces encountered during accelerations. In particular, the present study is motivated by the diversity of initial conditions that may be encountered at the onset of acceleration. We focus on the applicability of added-mass theory in separated flows and aim to understand the influence of initial conditions on the added-mass coefficient. This is accomplished by comparing forces measured experimentally and from simulations to those predicted by potential-flow theory for a canonical test case. We decompose the instantaneous drag forces exerted on a circular flat plate for a range of accelerations including nearly impulsive motion, repeating the tests from rest and from 'steady-state' initial conditions. These two motions serve as extreme examples of kinematics encountered in nature and engineering, which severely violate assumptions of attached potential flow and therefore test the generality of added-mass force predictions. In addition, the circular flat-plate geometry is dominated by flow separation and has a known analytic potential-flow added-mass coefficient. It therefore provides a simple framework with which to systematically investigate the forces arising from different initial wake conditions. A number of previous studies have also focused on the circular flat plate as a benchmark test case - see for instance Tao and Thiagarajan $(2003 \mathrm{a}, \mathrm{b})$ who investigated the vortex dynamics and resulting hydrodynamic forces on oscillating circular plates - which further increases the utility of the circular flat plate as a baseline geometry for continued characterization. While the results presented herein focus on the circular flat-plate geometry alone, the insight can be extended to more complex bodies subjected to flow separation during acceleratory motions. The presentation of the manuscript is as follows. In $\S 2$, the theoretical addedmass coefficient for a circular flat plate is discussed, followed by an outline of the experimental and numerical methods in $\S 3$ and $\S 4$. Vortex evolution in the wake of the plate and the instantaneous drag forces are investigated in $\S 5$. Finally, the main conclusions of the study are summarized in $\S 6$.

\section{The inviscid concept of added mass}

The added-mass force can be solved algebraically for a variety of body shapes using potential theory once the time-varying velocity field is known. For a body translating at a constant speed $(U)$ through a fluid, the kinetic energy of the body and surrounding fluid will also be constant, and proportional to the velocity squared. If the body begins to accelerate, then the kinetic energy in the surrounding fluid will increase. The time rate of change 
of the kinetic energy can be written in terms of the additional work that must be done by the body to increase the kinetic energy of the fluid (Brennen, 1982). For a circular flat plate accelerating normal to the fluid, the resulting added-mass force experienced by the body can be expressed as:

$$
F_{A M}=\frac{1}{3} \rho D^{3} a,
$$

where $F_{A M}$ is the added-mass force, $\rho$ is the fluid density, $D$ is the diameter of the plate, and $a$ is the acceleration (Lamb, 1932). This equation can be arranged into the two $\Pi$-groups for force and acceleration as follows:

$$
C_{a}=\frac{1}{3}=\frac{F_{A M}}{\rho D^{3} a}=\left(\frac{F_{A M}}{\rho D^{2} U^{2}}\right)\left(\frac{U^{2}}{a D}\right),
$$

where $C_{a}$ denotes the added-mass coefficient, and has a value of $1 / 3$ for a translating circular flat plate. The inverse of the second dimensionless group is known as the acceleration modulus, defined as $a^{*}=a D / U^{2}$. Therefore, the normalized ratio of the added-mass force and the acceleration modulus returns the added-mass coefficient. Note that for a circular plate the definition of the drag coefficient is simply a scaled form of the force normalization given above:

$$
C_{d}=\left(\frac{F}{\rho D^{2} U^{2}}\right)\left(\frac{8}{\pi}\right) .
$$

It should be noted here that the $\Pi$-groups shown in equation 2 and 3 are used to normalize the force data from the experiments and numerical simulations.

Figure 1(a) provides qualitative streamline trajectories corresponding to the potential flow solution, from which the added-mass coefficient is derived, near the body of an accelerating circular flat plate. In a real, viscous flow it is uncertain whether the same value for the added-mass coefficient is preserved during accelerations, and if so, for what conditions it is satisfied. The effect of flow separation on the added-mass coefficient during accelerations from rest (hereafter referred to as case 1 or $C 1$ ) and non-zero initial velocities (referred to as case 2 or $C 2$ ) is to be determined in this study. These two cases are visually depicted in figures 1(b) and (c), respectively. Although when the plate is accelerated from rest $(C 1)$ the flow will instantaneously separate at the plate edge, one would expect the subsequent development of the velocity field to vary when compared to $C 2$, thereby resulting in different 

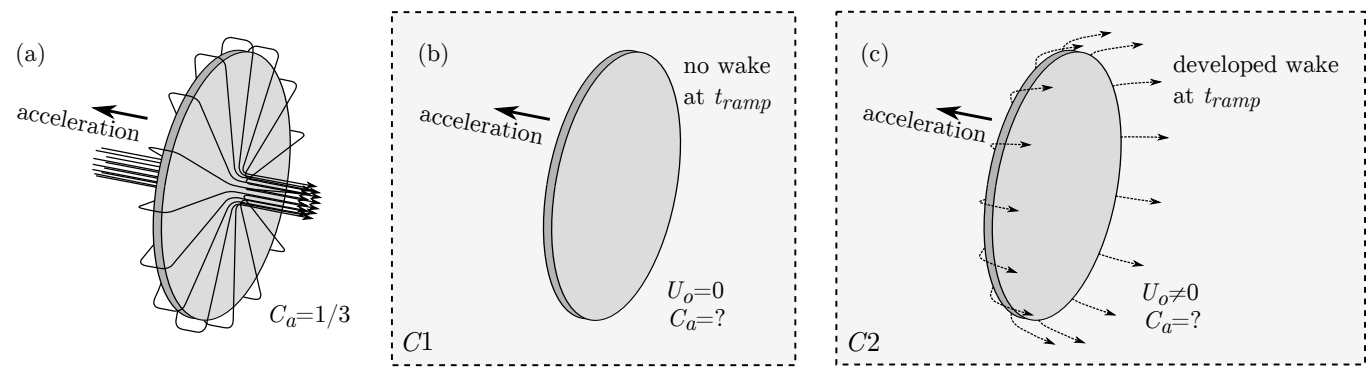

Figure 1: (a) Representative streamlines near the body of an accelerating circular flat plate (potential-flow solution). The potential-flow solution yields an added-mass coefficient of $8 / 3$. The effect of wake vorticity on the added-mass coefficient is investigated experimentally during accelerations (b) from rest, $C 1$, and (c) non-zero initial velocities, $C 2$.

time-force histories as well. This proposition is explored using the numerical and experimental data in $\S 5$.

\section{Experimental methods}

A circular flat plate with a diameter of $0.3 \mathrm{~m}$ and thickness of $3 \mathrm{~mm}$ was investigated in this study. All experiments were performed in an optical towing-tank facility at Queen's University, which has a cross-section of $1 \mathrm{~m}$ $\times 1 \mathrm{~m}$ and a total length of $15 \mathrm{~m}$. The towing tank is equipped with a roof that runs along the entire length of the apparatus to minimize free-surface effects. Fernando and Rival (2016a) previously showed that positioning of the circular plate at the center of the tank results in negligible blockage effects.

The plate underwent motions consisting of an acceleration from rest to the first steady velocity, $U_{1}$, followed by a second acceleration to the final steady velocity, $U_{2}$, before decelerating back to rest. The motions were normalized using the following relationship:

$$
t^{*}=\frac{t U_{1}}{D}
$$

where $t$ is the physical time of the motion, $U_{1}$ is the first steady velocity, and $D$ is the diameter of the plate. A second parameter, $s^{*}$, which represents the normalized distance travelled by the plate, was used to compare vortex growth behind the plate for different accelerations. This parameter is defined as: 


$$
s^{*}=\frac{s}{D},
$$

where $s$ is the physical distance travelled, and $D$ is defined as above. A normalized distance was used in lieu of a normalized time for comparisons between accelerations, since for varying accelerations, the plate will have travelled different distances over the same $t^{*}$ interval. Previous studies, Taneda and Honji (1971) for instance, have shown that vortex growth scales best with distance travelled rather than elapsed time.

The added-mass coefficient was investigated by maintaining the initial and final velocities for all motions in the parameter space, while varying only the acceleration period of both ramps. Eight different acceleration moduli were investigated. The corresponding motions are plotted in figure 2 as velocity normalized by the first steady velocity against dimensionless time. Note that the curves shown in figure 2 represent the commanded motions; a sample comparison between the commanded motion and the actual motion of the plate can be found in Appendix A for the acceleration case $a^{*}=0.333$. Initial and final Reynolds numbers of 125000 and 250000 , respectively, were maintained for all motions. An $a^{*}$ range between 0.333 and 4 was possible with the current experimental set-up. The exact $a^{*}$ values tested are listed in figure 2 , along with the equivalent $t^{*}$ values. Note that equation 4 can be used to appropriately scale motions with different values for $U_{1}$ and $U_{2}$. Although varying values for the final velocities would yield different values of $a^{*}$, these cases would simply occupy different regions of the curve shown in figure 2.

Analysis of existing circular flat-plate force data from Fernando and Rival (2016b) was used to inform the choice of ramp locations in the current study. The plate was accelerated to the second steady velocity at $t^{*}=24$, once the steady-state drag of the plate was established, as illustrated in figure 2 . The same ramp location was also used in the simulations.

\subsection{Force and particle image velocimetry measurements}

The plate was towed normal to the flow via a horizontal, cylindrical sting, which has a diameter of $0.08 D$ and a nominal length of $2 D$. A section of the towing tank (approximately $3 \mathrm{~m}$ in length) is depicted in figure 3(a), along with the mounting configuration of the plate. The black arrow indicates the towing direction of the plate. A six-component, submersible ATI Nano25 IP68 force transducer was mounted on the leeward side of the plates to record 


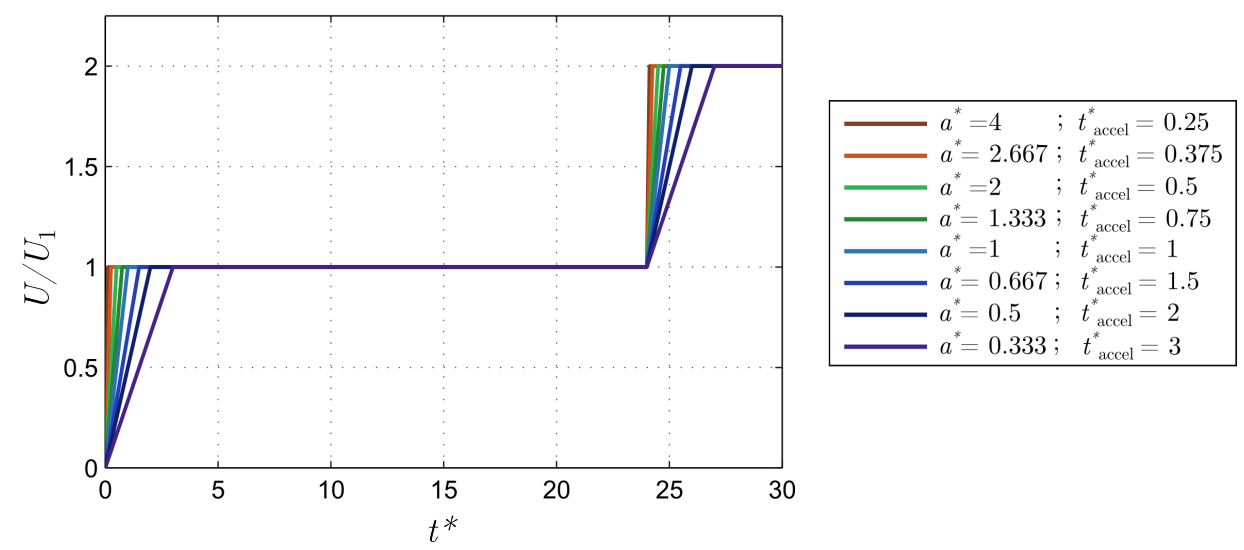

Figure 2: The eight motions investigated with acceleration moduli between $a^{*}=0.333$ and 4. The ramp to the final velocity $\left(U_{2}\right)$ was prescribed at $t^{*}=24$, once the steady-state drag force had been achieved.

force data, as shown in the inset in figure 3(a). The transducer has a static resolution of $0.125 \mathrm{~N}$ and was operated at a sampling rate of $1000 \mathrm{~Hz}$. All data sets were averaged over 20 runs. The standard deviation of the measured forces for each case shown in $\$ 5$ (figure 5) can be found in Appendix B. In addition, three unfiltered force-history plots for the highest and lowest acceleration cases $\left(a^{*}=0.333\right.$ and 4$)$ are shown in Appendix $\mathrm{C}$ to illustrate the repeatability of the experiments. Pictures of the physical set-up are provided in Appendix D.

Time-resolved, planar particle image velocimetry (PIV) was used to measure vortex growth in the wake of the plate for both cases. A right-hand coordinate system was adopted such that the $x$-, $y$-, and $z$-axes are oriented in the streamwise, wall-normal, and spanwise directions, respectively. The flow-field was captured using a Photron SA4 high-speed camera, which has a resolution of $1024 \times 1024$ pixels, and was operated at frame rates of 500 and $1000 \mathrm{~Hz}$. A $40 \mathrm{~mJ}$ pulse $^{-1}$ Photonics high-speed laser was used to create a laser sheet approximately $1.5 \mathrm{~mm}$ thick. The field of view (FoV) used for all measurements was approximately $1.2 D \times 1.2 D$ in size and was situated at the horizontal midspan of the plate (i.e. in the $x y$-plane) along the lower half of the sting, as shown in figure $3(\mathrm{~b})$. The towing motions were repeated and recorded 20 times for each FoV, and the results were phase-averaged. 


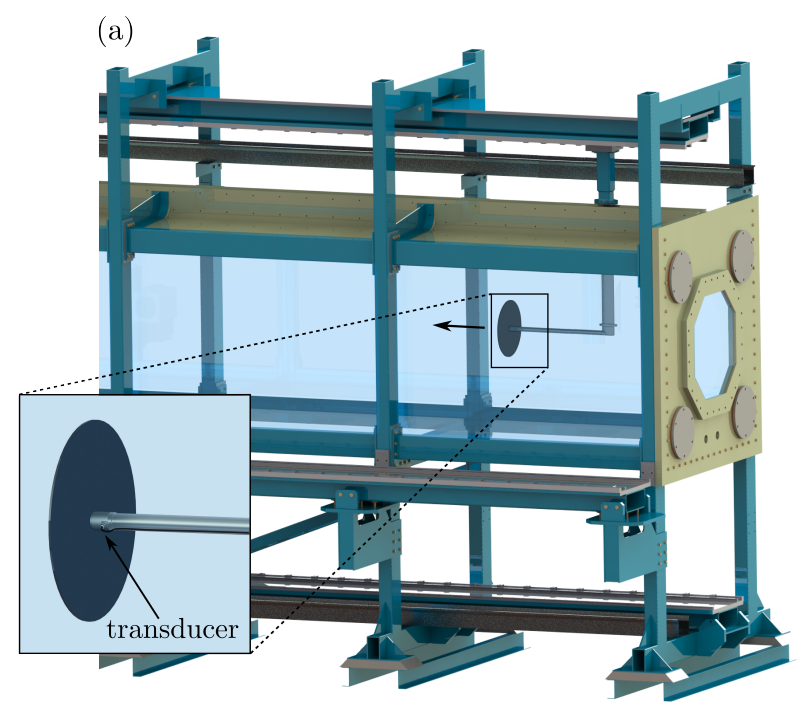

(b)

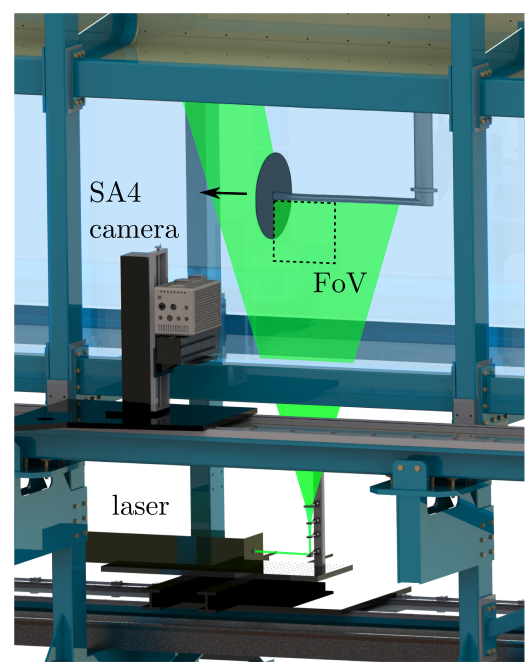

Figure 3: (a) Towing-tank facility depicting the circular flat plate at the beginning of the prescribed motion, and the location of the force transducer (shown in the inset); (b) the experimental set-up for PIV measurements, including the location of the high-speed camera and laser, and the approximate size and orientation of the field of view (FoV).

\section{Numerical method}

A set of fully three-dimensional Navier-Stokes simulations are used to provide an additional set of high-resolution field and force data, as well as to explore acceleration values that are not achievable experimentally.

The physical system is the same as the experiments except that the flow around the disk geometry was simulated without the sting. The simulated domain size matched the experimental tank cross-section, and the velocity profile shown in figure 2 was used in the simulations as well. Simulations across the acceleration range were run for two sets of final Reynolds numbers: $R e=2000$ and $R e=250000$ (with initial Reynolds numbers of $R e=1000$ and $R e=125000$, respectively). This broad Reynolds-number range, which is not achievable with the experimental set-up, was used to determine the sensitivity of the computed forces to Reynolds number, and thereby illustrate whether viscous effects are significant.

The simulations use the boundary data immersion method (BDIM) (Weymouth and Yue, 2011; Maertens and Weymouth, 2015), a robust immersed boundary method suitable for dynamic fluid-structure-interaction (FSI) problems. Briefly, BDIM solves for the fluid velocity and pressure on a 3D Carte- 
sian background grid by convolving the Navier-Stokes equations with the solid body equations of motion - in this case, the prescribed rigid body motion detailed above. The code uses an implicit-LES method for subgrid scale modelling (Maertens and Weymouth, 2015), which deactivates when the flow features are adequately resolved by the grid. Previous work has experimentally validated this approach for a variety of unsteady motion problems including accelerating foils and plates (Polet et al., 2015; Weymouth et al., 2007). The method has also been validated for fully detached threedimensional turbulent wakes in the same Reynolds number range as the current study (Garcia-Font et al., 2019).

No-slip and no-penetration boundary conditions are imposed on the solidfluid interface and a no-penetration condition is used on the domain boundaries. A uniform grid is used in the near wake region to resolve the thin shear layer as well as the vorticity separation and evolution. Exponential grid stretching is applied in the far-field. A numerical convergence study was carried out, varying the near wall grid spacing on the face of the disk $h$ from $D / 64$ to $D / 512$. We observed approximately second order convergence in the peak forces $(p=2.21)$ with a difference of less than $2 \%$ for $h=D / 256$ and $D / 512$. We chose to use the finest grid for the remainder of the work.

\section{Results and discussion}

In $§ 5.1$ PIV measurements and simulated data are first presented for $C 1$ and $C 2$ to compare the evolution of the vorticity fields between both cases and qualify the wake topology between cases. Next in $\$ 5.2$, instantaneous force data from the experiments for the eight $a^{*}$ values are presented, and the peak added-mass forces are compared to those extracted from the simulations and calculated from potential theory. The residual forces are then discussed at the end of this section.

\subsection{Vortex topology}

Velocity-field data are presented in figure 4 as vorticity contour plots from the experiments and simulations. Vorticity contours are provided at only a single cross-section (the horizontal midspan) for various dimensionless distances, since the vortex ring remains axisymmetric during the growth phase, as shown by Fernando and Rival (2016b). These data corresponds to an intermediary acceleration value $\left(a^{*}=1\right)$ and provides a sense of how similar the wake topology is between the experiments and simulations. The strong 
similarities between the two data sets, discussed subsequently, support the use of the simulations to understand the forces at large values of $a^{*}$ that were not possible experimentally. Vorticity is normalized by $\omega^{*}=\omega D / U_{f}$, where $U_{f}$ is specified as $U_{1}$ or $U_{2}$ depending on the case.

In general, the experimental data and simulations show almost identical topological trends, with similar magnitudes of vorticity. When the plate is accelerated from rest, a vortex ring rolls up in the wake of the plate, as shown in the timestep at $s^{*}=0.5$. In the second timestep for this case, the approximate distance between the center of the primary vortex and the plate is nearly identical in the two data sets $(\sim 0.3 D)$, as annotated in the figures in the second column from the left. When the vortex ring breaks down the steady wake is established, with a vortex sheet continually being shed from the plate edge. As the plate is accelerated from this state, the shear layer rolls up, and a second vortex ring is formed. The instantaneous velocity increase results in a shallower shear-layer angle, and the vortex ring evolves further away from the plate as it grows. As such, a larger distance between the plate and vortex center is observed $(\sim 0.43 D)$ in both the PIV and simulations.

The wake topology is different enough between the two cases, particularly with regards to the approximate distance of the vortex center-of-mass from the plate, that one would expect to see noticeable differences in the total forces. In the following subsection, the instantaneous drag forces are discussed.

\subsection{Instantaneous drag forces}

The experimental force histories for the eight accelerations outlined in $\S 3$ are shown in figure 5 as normalized force against time (see equation 2 ). Note that when accelerating from rest $(C 1)$, rather than immediately rising to finite values at the onset of motion, the forces increase continuously from zero, since an infinite jerk cannot be achieved experimentally. Similarly, when the accelerations are imposed from $U_{1}(C 2)$, the forces increase gradually from the steady-state value. The force peaks, which coincide with the end of the acceleration periods, are indicated by the black arrows. These peaks are isolated and compared to values obtained from the larger simulation parameter space, and to the added-mass forces predicted by potential theory. A comparison of the experimental and simulation force histories for a few acceleration cases is provided in Appendix E.

Figure 6 provides this comparison for the two cases separately. The force peaks are again normalized as in equation 2 (using $U_{1}$ in both cases as the 

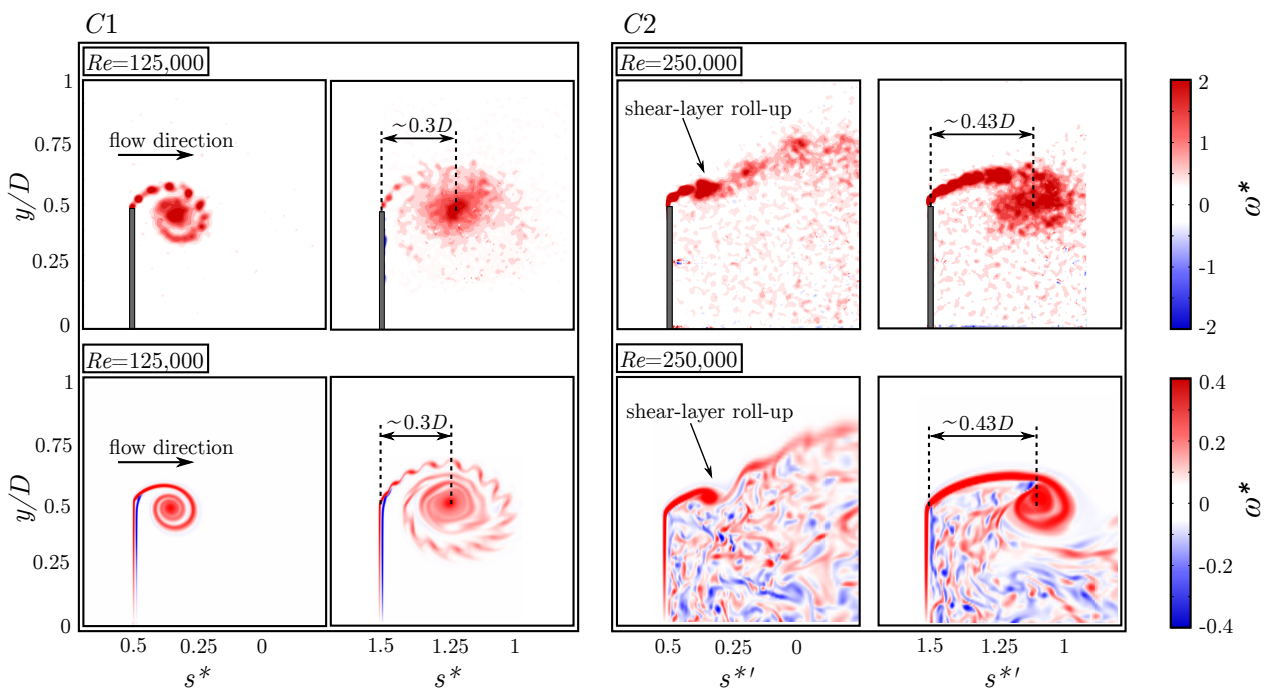

Figure 4: Vorticity fields from PIV measurements (top row) and simulations (bottom row). The experimental and simulation data corresponds to initial $(C 1)$ and final $(C 2)$ Reynolds numbers of $R e=125000$ and 250000 . Case $1(C 1)$ is shown in the first two columns at timesteps of $s^{*}=0.5$ and 1.5 , and case $2(C 2)$ is shown in the last two columns at $s^{* \prime}=0.5$ and 1.5 , where $s^{* \prime}=0$ represents the start of the second acceleration. The experimental results and simulations show good agreement with one another at equivalent time steps. When the plate is accelerated from rest, a vortex ring forms and evolves behind the plate while remaining in close proximity. At $s^{*}=1.5$, the center of mass of the vortex shows good collapse between the PIV and simulations. Once the vortex ring breaks down, the steady-state wake configuration is achieved, and the plate is accelerated again. The instantaneous velocity increase reduces the shear-layer angle, and the second vortex ring that forms convects further away from the plate during growth. 


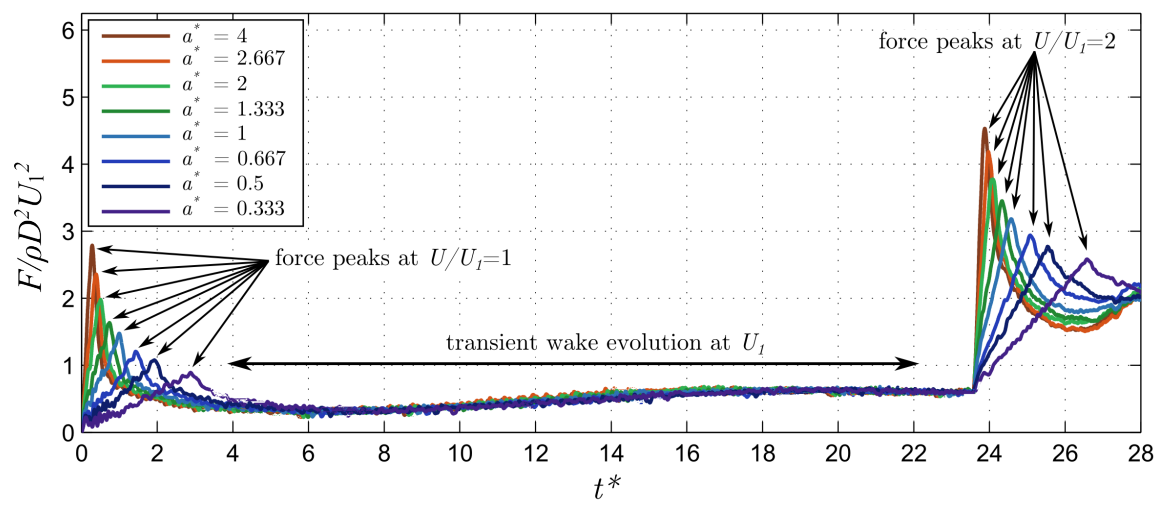

Figure 5: Experimental drag-coefficient histories plotted against dimensionless time for the eight acceleration cases. Maxima in the force histories coincide with the end of the acceleration period from rest to $U_{1}$ (case 1 ) and from $U_{1}$ to $U_{2}$ (case 2).

characteristic velocity to match the data shown in figure 5), and are plotted against the acceleration moduli. The added-mass forces $\left(F_{A M}\right)$ calculated from potential theory are indicated by the black line. Much like the vorticity-field comparisons, the experimental data and simulations show excellent collapse over the common $a^{*}$ range. A maximum discrepancy of $6 \%$ is observed between the experimental and simulated data sets, which further strengthens the use of the simulations to understand the forces at larger values of $a^{*}$. Note that the extensive simulation data set shown in figure 6 was calculated for the lower Reynolds number case due to computational cost. However, the force peaks for three acceleration values $\left(a^{*}=0.5,2\right.$, and 8$)$ at the higher Reynolds numbers were also calculated for $C 1$ and $C 2$ to ensure Reynolds-number independence in the results. A maximum of $4 \%$ difference between the low- and high-Reynolds number cases was calculated.

Past an acceleration value of approximately $a^{*}=4$, the slope of the forcepeak curve for both cases is almost identical to that of the added-mass coefficient - see figure 6(a) and (b). To understand the bias between the two curves, we consider the additional effects that contribute to the total drag force. During accelerations, in addition to the added-mass forces, there will also be a drag force associated with the instantaneous plate velocity (Brennen, 1982). Since the force peaks $\left(F_{\text {peak }}\right)$ occur at the end of the acceleration period when the plate has reached the final velocity, the steady drag force $\left(F_{s s}\right)$ has also been included in figures $6(\mathrm{a})$ and $(\mathrm{b})$ as the red lines. The steady drag force occurs once a steady wake has developed behind the plate, 
shown for $C 1$ in figure 5 between $t^{*}=20-23$. For $C 2$ this value is scaled by $U_{2}^{2} / U_{1}^{2}$. What remains are the vortex forces resulting for the formation and growth of the vortex rings behind the plate. Note that the magnitudes of the measured steady-state drag forces $\left(F_{s s}\right)$ for $C 1$ and $C 2$ are in good agreement with values reported in the literature for circular flat plates; see for instance Roos and Willmarth (1971) and Shenoy and Kleinstreuer (2008).

To characterize the overall contribution of the remaining drag effects, a residual drag force can be calculated. The residual is expressed as a fraction of the total peak force through the following relationship:

$$
F_{R}{ }^{*}=\left(\frac{\left(F_{\text {peak }}-F_{A M}-F_{s s}\right)}{\rho D^{2} U_{f}^{2}}\right)\left(\frac{8}{\pi}\right),
$$

where each force term in the equation is given in figures $6(\mathrm{a})$ and (b). The drag residual, $F_{R}{ }^{*}$, is plotted for $C 1$ and $C 2$ in figures $6(\mathrm{c})$ and (d) using the force peaks from the simulations. For $C 2$, the acceleration is imposed from a non-zero initial velocity, and so the plate will have travelled three times the distance — when compared to $C 1$ - by the end of the acceleration. It is reasonable to expect different magnitudes between the two cases, since the wake has evolved over different physical distances at the instant the force peak is extracted. Figures $6(\mathrm{c})$ and (d) indicate that there is only a noticeable discrepancy in the residuals between $C 1$ and $C 2$ for low accelerations, with the residual fraction approaching a value of approximately $20 \%$ in both cases with increasing acceleration.

To qualify the wake development, three insets of the vorticity field from the simulations have been superimposed on figures $6(\mathrm{c})$ and (d) for roughly evenly spaced $a^{*}$ values of $0.5,4$, and 8 . At $a^{*}=0.5$ for $C 1$, the leftmost inset in figure $6(\mathrm{c})$, the vortex ring has evolved over two diameters of travel, and the vorticity has already redistributed over a large area in the wake. In contrast, at $a^{*}=4$ and 8 , the vortex rings are fed the full-strength shear layer over less than half a diameter in each case and the vorticity is concentrated near the plate. For the insets corresponding to $C 2$, shown in figure $6(\mathrm{~d})$, the development of the wake for the slowest acceleration reveals a less coherent vortical structure, but with a similar trend of significant vorticity redistribution in the wake, which is topologically very similar to the steady-plate wake. Although the shear-layer roll-up for $a^{*}=4$ and 8 is evident in the vorticity fields, the overall distribution of vorticity in the wake is comparable to 

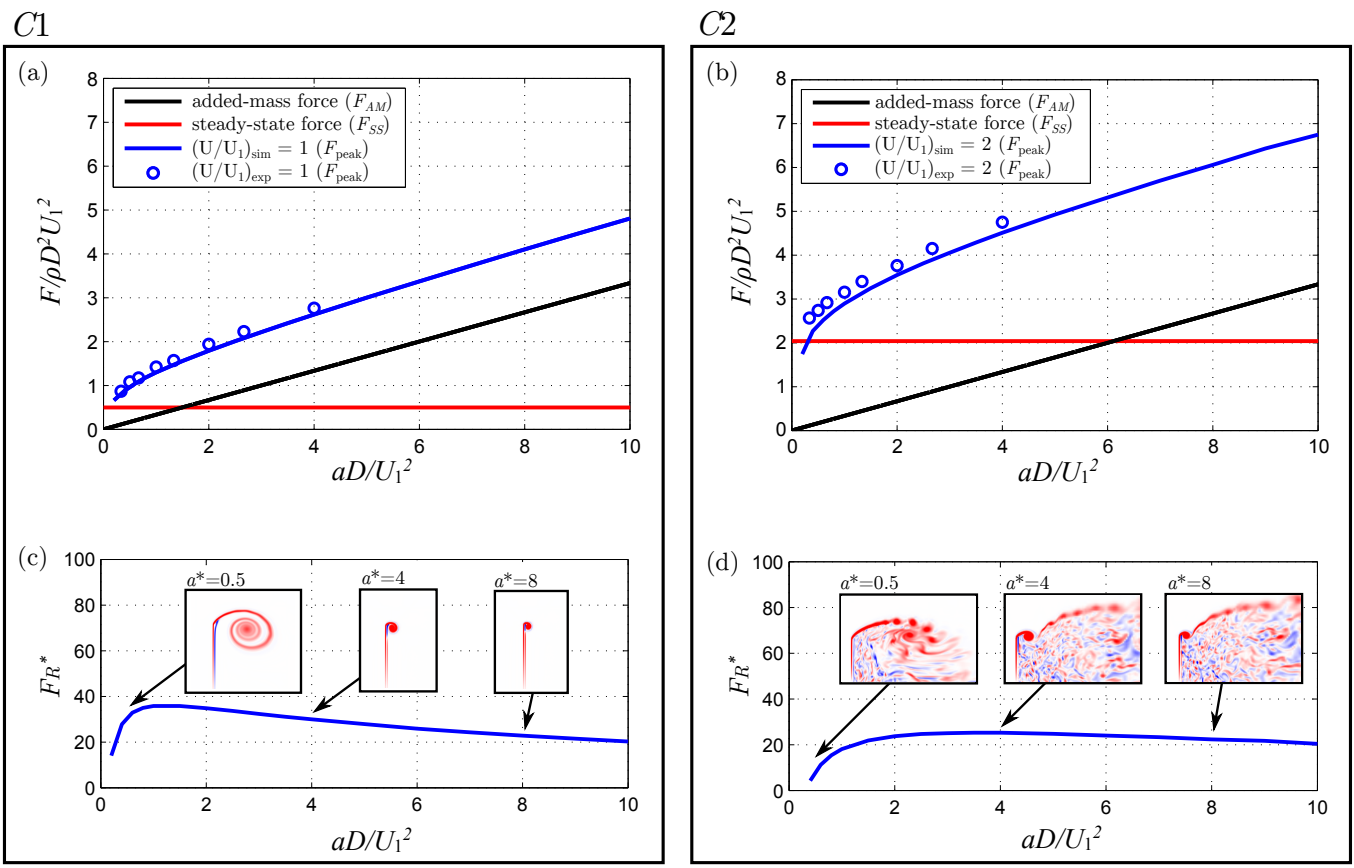

Figure 6: (a) Normalized force peaks at $U / U_{1}=1$ from the experimental data shown in figure 5 (blue hollow circles) and at $U / U_{1}=1$ (blue solid line) from the simulations. The simulation data is for the $R e=2000$ case, however, the normalized forces showed the same behaviour at either Reynolds number over the acceleration parameter space. The slope of the black line represents the value of the added-mass coefficient from the potential flow solution, and the red line represents the (constant) steady-state force. (b) Normalized force peaks from experimental data and simulations at $U / U_{1}=2$, along with the addedmass and steady-state force curves. The experimental data and simulations show strong collapse in (a) and (b), and similar trends are observed in both cases. Past an acceleration of $a^{*}=2$, a constant slope is observed in the force peaks. The added-mass and steady-state forces are subtracted from the force peaks to yield the residual force coefficient plotted in (c) for $C 1$, and (d) for $C 2$. Insets of the vorticity field are provided for $a^{*}$ values of $0.5,4$, and 8 in both cases to highlight the state of the wake with increasing acceleration. 
that of the steady-state wake, and the force residuals are of a comparative magnitude to the lower acceleration case.

\section{Conclusions}

In this study, the forces exerted on a circular flat plate have been investigated using experimental measurements and simulations of the viscous Navier-Stokes equation. In particular, we investigated whether the inviscid concept of added mass is sufficient to capture the force on accelerating bodies in highly-separated flows. To this end, the circular flat plate was tested over a large range of accelerations, which were imposed on the plate both from rest and from a non-zero initial velocity. The theoretical added-mass forces and steady-state forces were subtracted from the peak values at the end of the accelerations to calculate the residual forces. The results can be summarized in two main points:

1. The residual forces during accelerations from both zero and non-zero initial velocities yield strong collapse with respect to one another, particularly for large accelerations (i.e. in the limit of impulsive motions). This indicates that the total forces are not a strong function of the initial state of the wake; and

2. Even in the limit of impulsive motions, for which the potential-flow solution should be the most viable, the residual forces are not driven to zero, but instead asymptote to $20 \%$ of the total reactant force for both initial conditions.

Therefore, we are led to two surprising conclusions; that force predictions with fully-separated flows may be made without detailed knowledge of the initial state of the wake, and that inviscid added-mass forces do not alone account for the total forces during impulsive motions.

Although only a single geometry was investigated in this study, the circular flat plate epitomizes the most severe cases of flow separation, and therefore the results presented may be transferable to other similarly complex cases where it is desirable to quickly and accurately estimate the unsteady forces.

\section{Acknowledgements}

The authors acknowledge the support from the Natural Sciences and Engineering Research Council of Canada. 


\section{References}

Babinsky, H., Stevens, P., Jones, A., Bernal, L., Ol, M., 2016. Low order modeling of lift forces for unsteady pitching and surging wings. In: 54th AIAA Aerospace Sciences Meeting. p. 0290.

Baik, Y., Bernal, L., Granlund, K., Ol, M., 2012. Unsteady force generation and vortex dynamics of pitching and plunging aerofoils. Journal of Fluid Mechanics 709, 37-68.

Brennen, C., 1982. A review of added mass and fluid inertial forces. Technical Report: Department of the Navy.

Clift, R., Grace, J., Weber, M., 1978. Bubbles, drops, and particles. Academic Press.

Fernando, J., Rival, D., 2016a. On vortex evolution in the wake of axisymmetric and non-axisymmetric low-aspect-ratio accelerating plates. Physics of Fluids 28 (1), 017102.

Fernando, J., Rival, D., 2016b. Reynolds-number scaling of vortex pinch-off on low-aspect-ratio propulsors. Journal of Fluid Mechanics 799, R3.

Garcia-Font, B., Weymouth, G., Nguyen, V., Tutty, O., 2019. Span effect on the turbulence nature of flow past a circular cylinder. Journal of Fluid Mechanics 878, 306-323.

Karanfilian, S., Kotas, T., 1978. Drag on a sphere in unsteady motion in a liquid at rest. Journal of Fluid Mechanics 87 (01), 85-96.

Küssner, H., 1936. Zusammenfassender bericht über den instationären auftrieb von flügeln. Luftfahrtforschung 13 (12), 410-424.

Lamb, H., 1932. Hydrodynamics. Cambridge university press.

Leonard, A., Roshko, A., 2001. Aspects of flow-induced vibration. Journal of Fluids and Structures 15 (3), 415-425.

Maertens, A., Weymouth, G., 2015. Accurate cartesian-grid simulations of near-body flows at intermediate reynolds numbers. Computer Methods in Applied Mechanics and Engineering 283, 106-129. 
Manar, F., Mancini, P., Mayo, D., Jones, A., 2015. Comparison of rotating and translating wings: force production and vortex characteristics. AIAA Journal 54 (2), 519-530.

Mancini, P., Manar, F., Granlund, K., Ol, M., Jones, A., 2015. Unsteady aerodynamic characteristics of a translating rigid wing at low reynolds number. Physics of Fluids 27 (12), 123102.

Odar, F., Hamilton, W., 1964. Forces on a sphere accelerating in a viscous fluid. Journal of Fluid Mechanics 18 (02), 302-314.

Pitt Ford, C., Babinsky, H., 2013. Lift and the leading-edge vortex. Journal of Fluid Mechanics 720, 280-313.

Polet, D., Rival, D., Weymouth, G., 2015. Unsteady dynamics of rapid perching manoeuvres. Journal of Fluid Mechanics 767, 323-341.

Prandtl, L., Tietjens, O., 1957. Applied hydro-and aeromechanics. Vol. 2. Courier Corporation.

Roos, F., Willmarth, W., 1971. Some experimental results on sphere and disk drag. AIAA journal 9 (2), 285-291.

Sarpkaya, T., Isaacson, M., 1981. Mechanics of wave forces on offshore structures.

Shenoy, A., Kleinstreuer, C., 2008. Flow over a thin circular disk at low to moderate reynolds numbers. Journal of Fluid Mechanics 605, 253-262.

Taneda, S., Honji, H., 1971. Unsteady flow past a flat plate normal to the direction of motion. Journal of the Physical Society of Japan 30 (1), 262272 .

Tao, L., Thiagarajan, K., 2003a. Low kc flow regimes of oscillating sharp edges i. vortex shedding observation. Applied ocean research 25 (1), 2135 .

Tao, L., Thiagarajan, K., 2003b. Low kc flow regimes of oscillating sharp edges. ii. hydrodynamic forces. Applied ocean research 25 (2), 53-62.

Theodorsen, T., 1935. General theory of aerodynamic instability and the mechanism of flutter. NACA Report 496, 2-21. 
Weymouth, G., Hendrickson, K., Yue, D., O'Shea, T., Dommermuth, D., Adams, P., Valenciano, M., 2007. Modeling breaking ship waves for design and analysis of naval vessels. In: DoD High Performance Computing Modernization Program Users Group Conference. IEEE, pp. 440-445.

Weymouth, G., Triantafyllou, M., 2012. Global vorticity shedding for a shrinking cylinder. Journal of Fluid Mechanics 702, 470-487.

Weymouth, G., Triantafyllou, M., 2013. Ultra-fast escape of a deformable jet-propelled body. Journal of Fluid Mechanics 721, 367-385.

Weymouth, G., Yue, D., 2011. Boundary data immersion method for cartesian-grid simulations of fluid-body interaction problems. Journal of Computational Physics 230 (16), 6233-6247. 


\section{Appendix A. Sample traverse motion comparison}

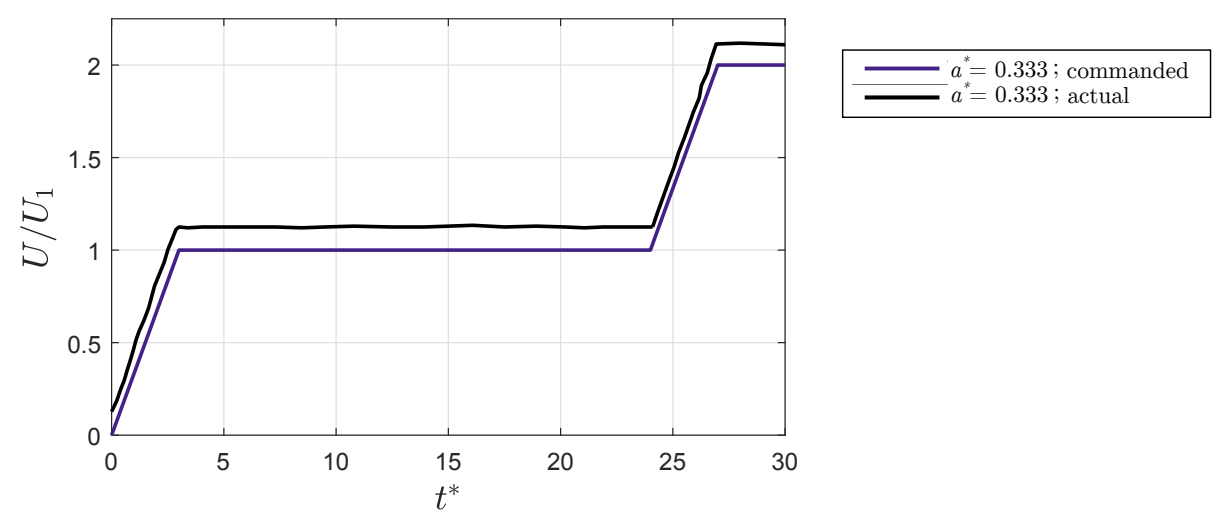

Figure A.7: Sample comparison between the time history of the commanded traverse motion (purple line) and the actual motion (black line) for the acceleration case $a^{*}=0.333$, as measured by LabView. Note that for the commanded motion, the y-axis data has been offset by $U / U_{1}=0.125$ for clarity.

\section{Appendix B. Standard deviation of force histories}

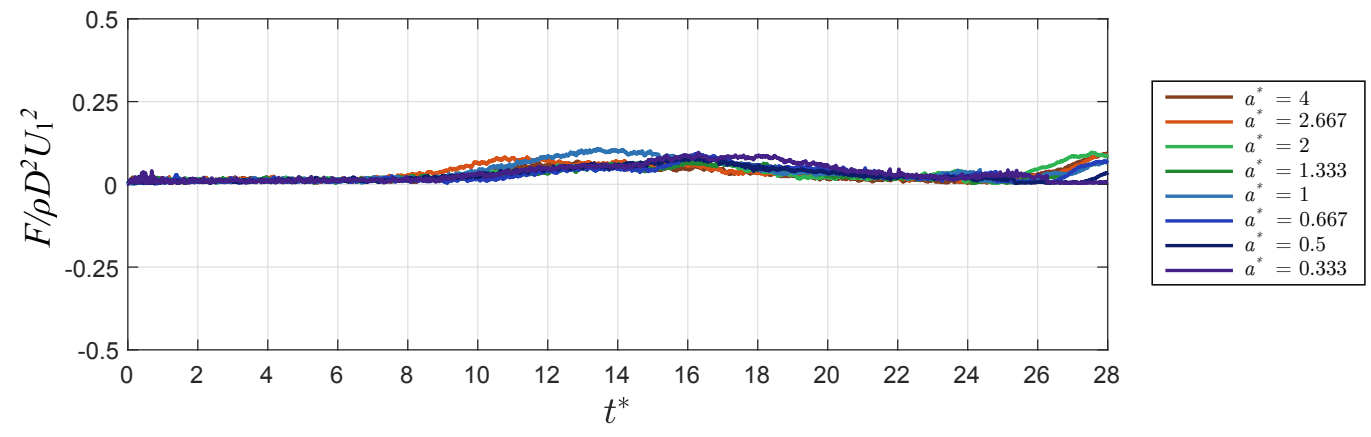

Figure B.8: Standard deviation of the force histories shown in Figure 5 for all eight acceleration cases. 


\section{Appendix C. Sample unfiltered force histories}
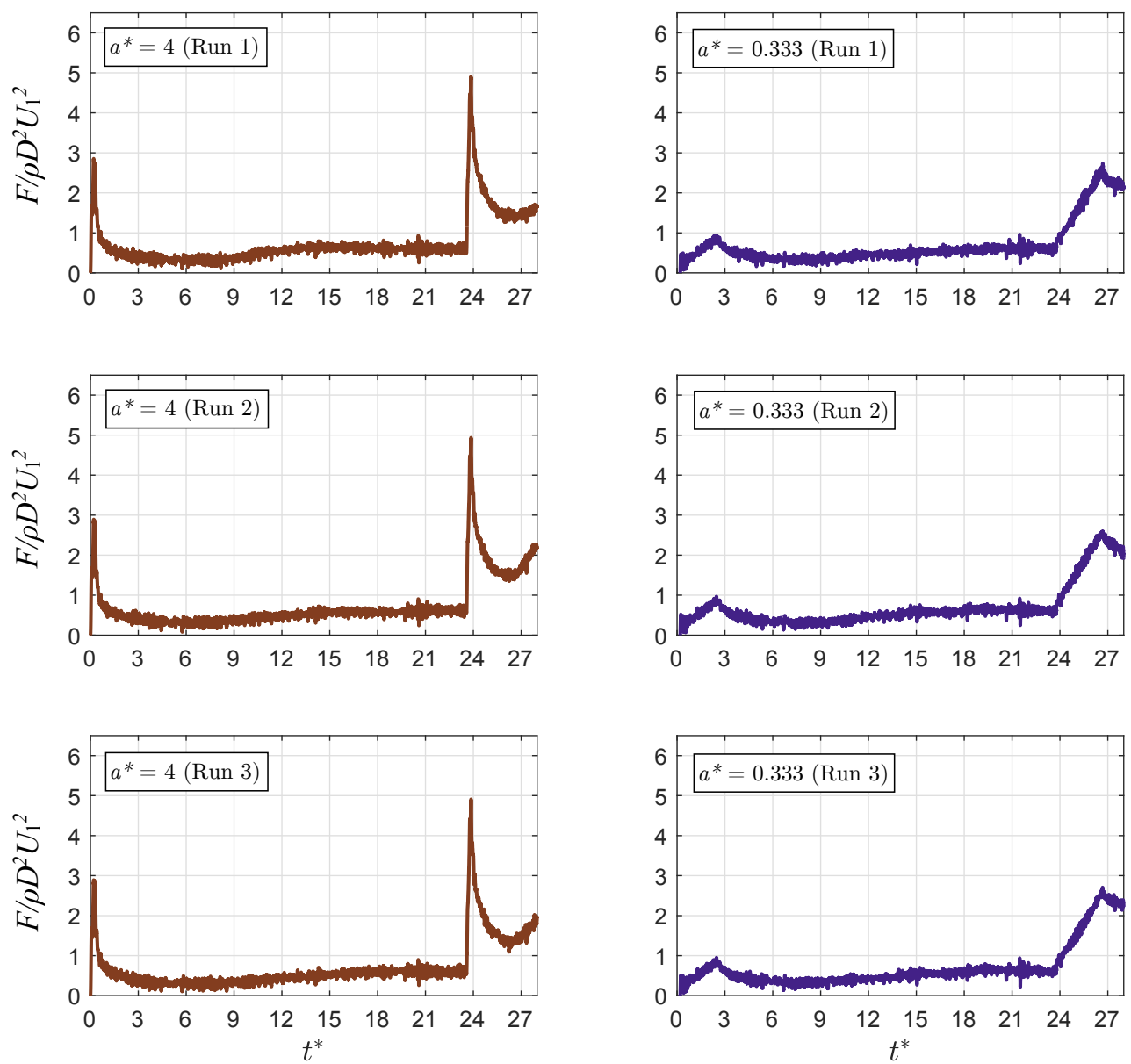

Figure C.9: Sample unfiltered forces histories for the fastest $\left(a^{*}=4\right)$ and slowest $\left(a^{*}=0.333\right)$ acceleration cases, shown in the left and right columns, respectively. Three runs are shown for each case, which are representative of all individual runs included in the force-history averages shown in Figure 5. 


\section{Appendix D. Experimental Set-up}

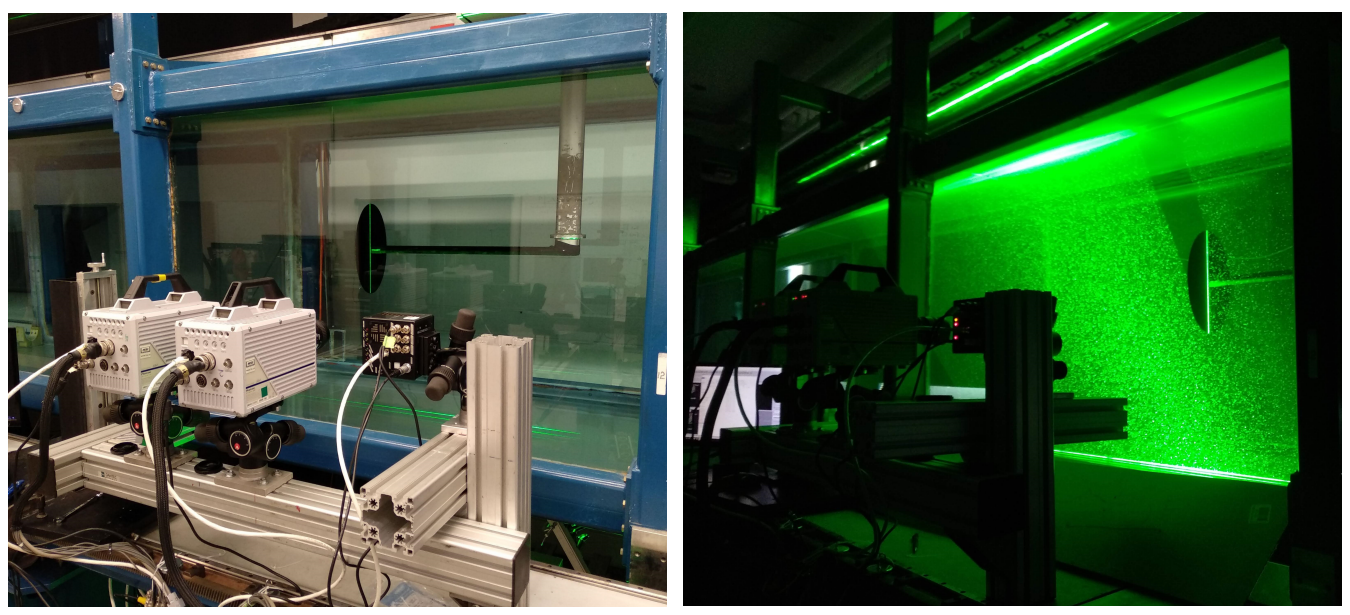

Figure D.10: Pictures of the experimental set-up showing the high-speed camera/test specimen in the towing tank (left) and the planar laser sheet (right) used for the PIV measurements. 


\section{Appendix E. Comparison of force histories}
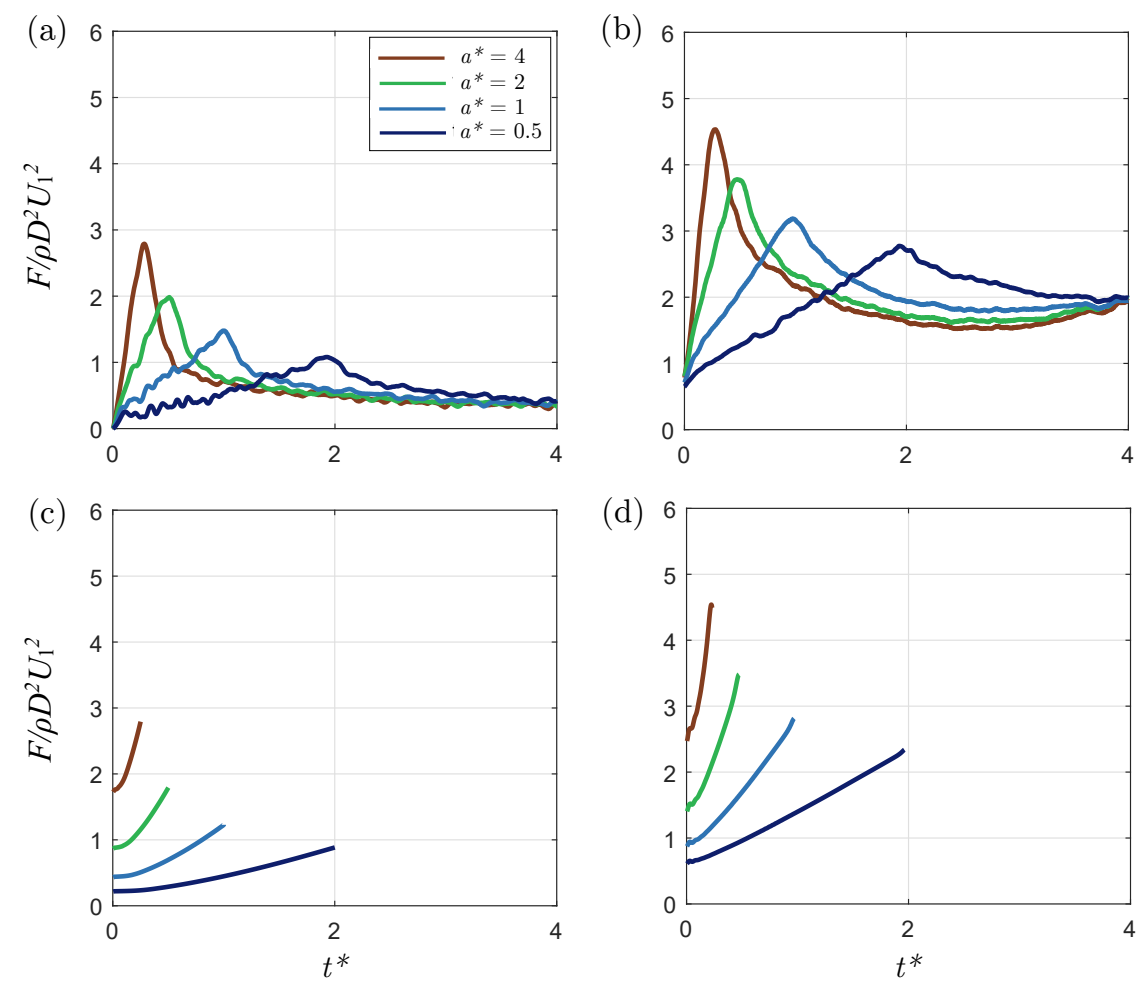

Figure E.11: Sample experimental force histories for four acceleration cases during accelerations from rest (a) and from constant velocities (b). Matching simulation forces histories for accelerations from rest (c) and constant velocities (d). Note that the objective of the simulations was to extract the force peak at the end of the accelerations, and therefore continuous force histories were only recorded until this point for a few acceleration cases to minimize computational cost. Note that the numerical simulations start with non-zero acceleration, so they immediately experience an added mass force. The experiments have some lag while the slack in the physical system is tightened. For this reason the force comparisons are based on the final peak velocity and acceleration, when the experimental set-up is operating at the ideal conditions. 